This thematic issue is dedicated to Kenneth D. Philipson, $\mathrm{PhD}$, Chair, Department of Physiology, University of California Los Angeles, on the occasion of his retirement.

\title{
Calcium is omnipresent in the physiology and pathophysiology of heart disease
}

\author{
Christian Pott, Lars Eckardt
}

Published online: 18 July 2012

(C) Springer-Verlag Wien 2012

In western industrialized nations, the burden of cardiovascular disease is increasing steadily. Despite numerous pharmacological, interventional, and surgical therapeutic options and promising novel additions to the field, cardiovascular disease remains the leading cause of death.

The physiology of the healthy human heart has been characterized well in vivo and in vitro. However, to develop novel therapeutic and diagnostic tools, it is necessary to transfer our physiological perceptions into the field of pathophysiology.

In human physiology, the role of calcium ions is ubiquitous. In particular, skeletal and cardiac muscles depend on calcium as their main effectors of contraction, but calcium is also essential in vascular function, neural transduction, endocrinological processes, and several other physiological functions of the human body. Similarly, the role of calcium is ubiquitous in the human heart. Not only it is an essential effector of the most obvious of all cardiac functions-contractility-but it also plays important roles in regulating and generating heart rhythm and metabolism. Thus, the contemplation of calcium handling in the development and maintenance of cardiac disease can of course not be limited to a single cardiac disease entity. Instead, one has to discuss the role of calcium in several cardiac disease categories such as arrhythmia, heart failure, and cardiac ischemia. One also has to be aware that these disease entities mutually depend on one another reciprocating their development and maintenance. In any of these three pathological entities-arrhythmia, heart failure, and ischemia-aberrations of calcium handling may not always be cause but can also be consequence of the disease mechanism in the sense of adaptive or maladaptive mechanisms. This thematic issue of the Wiener Medizinische Wochenschrift aims at adding to the translational research process by presenting a collection of papers that review specific cardiac disease entities in light of their associated aberrations of cardiac calcium handling. To begin with, Bögeholz et al. will give a brief overview on physiological calcium handling, i.e. as it occurs in the healthy human heart. Next, Frommeyer et al. and Heijman et al. are going to review the growing field of research on calcium handling in life threatening ventricular arrhythmias and atrial fibrillation, respectively. Closely related to this field, Kochhäuser et al. give an overview on mutations of calcium handling proteins and the associated disease categories. Reuter and Schwinger will review the topic of calcium handling in heart failure while Kleinbongard et al. give an overview on the role of calcium antagonists in ischemic heart disease. Finally, Vogler et al. will add a digest on established and novel pharmacological agents that can influence specific targets of cardiac calcium handling.

\section{Conflict of interest}

The authors declare no conflict of interest related to this study.

\footnotetext{
C. Pott, MD $(\varangle) \cdot$ L. Eckardt, MD, PhD

Division of Experimental and Clinical Electrophysiology, Department of Cardiology, University Hospital Münster, Albert-Schweitzer-Campus 1, 48149 Münster, Germany e-mail: christian.pott@ukmuenster.de
} 